\title{
Nanofriction mechanisms derived from the dependence of friction on load and sliding velocity from air to UHV on hydrophilic silicon
}

\author{
Andreas Opitz * $\dagger$ \\ Institut für Physik, Technische Universität Ilmenau, PF 100565, 98684 Ilmenau, \\ Germany \\ Syed Imad-Uddin Ahmed \\ CSEM Centre Suisse d'Electronique et de Microtechnique SA, Rue Jaquet Droz 1, \\ CH-2007 Neuchâtel, Switzerland \\ Matthias Scherge \\ IAVF Antriebstechnik AG, Im Schlehert 32, 76187 Karlsruhe, Germany \\ Juergen A. Schaefer \\ Institut für Physik and Zentrum für Mikro- und Nanotechnologien, Technische \\ Universität Ilmenau, PF 100565, 98684 Ilmenau, Germany
}

July 6,2021

\begin{abstract}
This paper examines friction as a function of the sliding velocity and applied normal load from air to UHV in a scanning force microscope (SFM) experiment in which a sharp silicon tip slides against a flat $\mathrm{Si}(100)$ sample. Under ambient conditions, both surfaces are covered by a native oxide, which is hydrophilic. During pump-down in the vacuum chamber housing the SFM, the behavior of friction as a function of the applied normal load and the sliding velocity undergoes a change. By analyzing these changes it is possible to identify three distinct friction regimes with corresponding contact properties: (a) friction dominated by the additional normal forces induced by capillarity due to the presence of thick water films, (b) higher drag force from ordering effects present in thin water layers and (c) low friction due to direct solid-solid contact for the sample with the counterbody. Depending on environmental conditions and the applied normal load, all three mechanisms may be present at one time. Their individual contributions can be identified by investigating the dependence of friction on the applied normal load as well as on the sliding velocity in different pressure regimes, thus providing information about nanoscale friction mechanisms.
\end{abstract}

Keywords: scanning force microscopy (SFM), nanotribology, nanofriction, silicon, silicon oxide, water film

\footnotetext{
* Corresponding author - email: andreas.opitz@physik.uni-ausgburg.de

† Present Address: Institut für Physik, Universität Augsburg, 86153 Augsburg, Germany
}

(c) 2021 Kluwer Academic Publishers. Printed in the Netherlands. 


\section{Introduction}

In microelectromechanical systems (MEMS), the motion of contacting parts is strongly influenced by the tribological properties of the system. Adhesion and friction play a critical role in determining the operational reliability and lifetimes of such systems [19]. One of the main causes of adhesion and friction problems of MEMS is the presence of a thin water film present on most surfaces. This is particularly true of silicon, the most popular of all the MEMS materials. Freshly exposed silicon rapidly oxidizes in air forming a nm thick hydrophilic oxide layer, which can ultimately dictate the performance of the entire device. Thus, it is important to thoroughly understand the adhesion and friction properties of contacting surfaces down to the micro and nanoscales. This knowledge will facilitate the fabrication of next generation micro and nanodevices with tailored tribological characteristics.

Previous investigations have examined different aspects of adhesion and friction properties of materials on the micro and nanoscale. The microfrictional properties of a tribosystem consisting of a $1 \mathrm{~mm}$ sapphire sphere sliding against a flat silicon sample was investigated from air to ultrahigh vacuum (UHV) $[16,17]$. This study showed that friction initially decreased exponentially as a function of the pump-down time. Later, as more water desorbed from the tribocontact distinct peaks with higher friction were seen in the friction versus pump-down time curve. These friction peaks were characterized by the occurrence of distinct stick/slip friction processes and regions between them that were absent from such effects. It was postulated that the peaks with stick/slip friction was caused by the solid-like friction of thin ordered water films confined between the ball and sample. It was further postulated that friction peaks occur whenever an ordering effect occurs due to the formation of a complete monolayer, which results in solidlike friction. In the transition period between complete monolayers, the molecules were judged to be not ordered. In this regime, friction was characterized only by small fluctuations and was liquid-like. Another group determined the coefficient of friction for two flat silicon samples [3] in a simple experiment. The lower sample was tilted until the upper sample just begins to slide. By recording the tilt angle, $\alpha$, when sliding occurred the coefficient of friction, $\mu$ was calculated from the relationship: $\mu=\tan \alpha$. The experiment showed that, when water coverage on the surface is reduced due to desorption in vacuum, friction is first reduced due to decreasing capillarity and is then followed by increased friction. Similar experimental results were obtained in experiments on single-crystalline $\mathrm{Ni}(100)$ [5] where friction was measured for decreasing thickness of an ethanol film in UHV. Smooth sliding at a coverage of 
about 300 monolayers changed into stick/slip sliding when the ethanol film was reduced to around a monolayer. This was then followed by strong sticking in the submonolayer range. The contact pressures in the above experiments were of the order of tens or at most hundreds of $\mathrm{MPa}$, which is not extremely high compared to most macro and even nano contact situations. Due to this characteristic, a liquid layer is present at all times on the surfaces between contacting parts and the confined liquid films are responsible for causing stick/slip effects etc. In contrast, in a typical SFM contact the contact area is much smaller $\left(\sim 20 \mathrm{~nm}^{2}\right)$. With a normal force on the order of $50 \mathrm{nN}$, the contact pressure comes out to be in the range of several GPa. This unique contact feature results in a tribological behavior that is different from the microfrictional studies discussed above.

In a nanotribological study, friction as a function of the sliding velocity, relative humidity and wettability was examined by Riedo et al. [15]. In this study, a linear dependence of friction force vs. logarithm of sliding velocity was observed. The slope for hydrophilic surfaces was determined to be negative and for higher sliding velocities the friction force decreased; the slope was reduced at lower humidities. The results were interpreted to indicate that the influence of capillary force is reduced with decreasing humidity and at higher sliding velocities. It was also shown that the slope for hydrophobic surfaces is positive and does not change with varying humidity.

A nanotribological investigation, in which the function of friction force and water film thickness vs. residual gas pressure during pump down in a vacuum chamber, was conducted by Opitz et al. [13]. In this report, different friction and contact regimes were identified. The water film thickness for hydrophilic native oxide covered silicon samples, measured by distance dynamic force spectroscopy, was found to reduce from about $2.6 \mathrm{~nm}$ to $0.7 \mathrm{~nm}$ with decreasing residual gas pressure in three distinct regimes. Further measurements showed the complete removal of water due to the combined effect of the vacuum environment and friction-induced desorption. The measured friction force also showed three regimes. It was seen that the reduction of the friction force corresponds to decrease of the water film thickness.

This work extends the knowledge gained in the above mentioned study [13] in that it examines the dependence of friction on velocity and on an externally applied normal load and uses this information to derive nanofriction mechanisms and basic nanocontact properties of the tribosystem. A continually decreasing water film thickness was produced by inserting the sample in a vacuum system and pumping it down from ambient pressure to $10^{-8}$ mbar. Hydrophilic, native oxide covered silicon was used as sample and counterbody. Distinct regimes 
of friction versus the applied normal load and the sliding velocity could be identified.

\section{Experimental setup}

\subsection{SAMPLE PREPARATION}

Rectangular pieces of p-doped (1-10 $\Omega \mathrm{cm}, \mathrm{B}$-doped) native oxide covered $\mathrm{Si}(100)$ were used as samples. These had an SFM determined RMS (Root Mean Square) roughness of about $0.1 \mathrm{~nm}$ measured over a $0.6 \times 0.6 \mu \mathrm{m}^{2}$ area. Due to the presence of a native oxide on top of the $\mathrm{Si}(100)$ surface, the samples are OH-terminated [14] and hydrophilic $[7,10]$. The hydrophilicity was also separately confirmed by contact angle measurements (not shown). Prior to insertion into the UHV chamber, the samples were sequentially cleaned using ultrasonic assistance in isopropanol and methanol for five minutes each. Afterwards, the samples were thoroughly rinsed with bi-distilled water. The oxide film measured on such samples has a thickness of $1.3 \mathrm{~nm}$ as determined by X-ray induced photoelectron spectroscopy (XPS).

\subsection{Friction FORCE MiCROSCOPE}

A UHV vacuum SFM/STM (Omicron Nanotechnology GmbH) system was operated in contact SFM mode to measure friction between a sharp silicon tip and a planar $\mathrm{Si}(100)$ sample. Measurements were performed at various stages during evacuation of the system, from air to $10^{-8}$ mbar, using a combination of turbo molecular, ion and titanium sublimation pumps. Starting from air, upon pump-down the chamber pressure decreased rapidly between $10^{1}$ and $10^{-3}$ mbar. Due to the fact that at this stage thermodynamic equilibrium does not exist, no measurements for the velocity and normal force characteristic could be performed. Friction measurements using contact SFM were performed in lateral mode registering the friction hysteresis at different applied normal loads with sliding velocity of $300 \mathrm{~nm} / \mathrm{s}$ and at different sliding velocities with an applied normal load of $60 \mathrm{nN}$. For each friction measurement the tip was scanned $300 \mathrm{~nm}$ in the forward and backward direction and the friction was determined by taking one half of the friction hysteresis curve [18]. To attain a high lateral force resolution, single beam Si cantilevers with a length of $440 \mu \mathrm{m}$ were chosen instead of triangular cantilevers that are typically used for topographical measurements. These single beam cantilevers, with a normal spring constant of $0.11 \mathrm{~N} / \mathrm{m}$, and a manufacture-quoted tip radius that was smaller than $15 \mathrm{~nm}$, were used as received, i.e. in 
an oxidized (hydrophilic) state. Calibration of the normal and lateral forces was achieved by following the procedure developed by Schwarz et al. [18], which consists of using the geometrical dimensions of the tip and determining the magnitude of tip deflection as a function of the applied load.

\section{Results}

\subsection{Residual gas PRessure Dependence}

Figure 1 shows the friction force measured as a function of the residual gas pressure from air down to $10^{-8} \mathrm{mbar}$. In this case, the silicon sample was cleaned as described above and then thoroughly rinsed with water before inserting it into the vacuum system. After this preparation process the hydrophilic sample is covered by a water with a thickness of $2 \mathrm{~nm}$. Initially, a high friction force was measured (Fig. 1). Afterwards, during pump-down, friction decreased from range (a) to range (b) in Fig. 1 to half the initial value. This is due to water desorption when the residual gas pressure is decreased. However, a subsequent reduction in the friction also occurred at lower pressures. It is suggested that this last step (marked (c) in Fig. 1) to lower friction values occurs due to the combined effect of the vacuum and friction-induced desorption of water from about $0.7 \mathrm{~nm}$ thickness to almost complete water removal. The resulting contact at this stage is then a direct solid-solid contact without the presence of an additional water film.

\subsection{Sliding Velocity DePendence}

The friction force vs. sliding velocity dependence, characteristic for the three ranges shown in Fig. 1, is depicted in Fig. 2. The sliding velocity is plotted in logarithmic scale. In this plot a linear dependence is visible. The equation used to fit this measurement is given as [15]

$$
F_{\mathrm{R}}=F_{\mathrm{O}}+F_{\mathrm{S}} \ln \left(\frac{v}{v_{0}}\right) .
$$

Here are $F_{\mathrm{O}}$ the intercept and $F_{\mathrm{S}}$ the slope. The constant $v_{0}$ was fixed to $1 \mathrm{~nm} / \mathrm{s}$ to keep the units correct.

Figure 3 shows the changing of the slope $F_{\mathrm{S}}$ as a function of the residual gas pressure. Like in Figure 1, here also the three distinct regions: (a), (b) and (c) can be easily discerned. The slope changes from high negative values for regime (a) to positive values in regime

(b) and ends at comparatively small positive values for regime (c). The 
boundaries between these regimes are the same like in the friction vs. residual gas pressure measurements (see Fig. 1).

\subsection{Normal ForCe DEPENDENCE}

Bowden and Tabor [1] described the dependence of friction force on the applied normal force with respect to the contact area. Later, Maugis [11] derived a value for the contact area from the elastic theory of solids and from rupture mechanics. In this work a parameter $\lambda$ is defined whose magnitude indicates which borderline case of the general contact model should be applied. It is defined as

$$
\lambda=\left(\frac{8 R \gamma^{2}}{\pi K^{2} \delta^{3}}\right)^{\frac{1}{3}}
$$

where $\gamma$ is the surface energy, $\delta$ the interaction length in the Dugdale interaction model, $R$ the tip radius and $K$ the reduced elastic modulus. Using the values $R=30 \mathrm{~nm}$ [4], $\gamma_{\mathrm{SiO}_{2}}=20 \mathrm{~mJ} \cdot \mathrm{m}^{-2}$ [10], $K_{\mathrm{SiO}_{2}}=50.1 \mathrm{GPa}[16]$ and assuming that the interatomic distance, $\delta$ is $0.5 \mathrm{~nm}$ the calculation for $\lambda$ gives 0.07 . When $\lambda$ is smaller than 0.1 the DMT-model [2] for the friction force $F_{\mathrm{R}}$ can be used:

$$
F_{\mathrm{R}}=\pi \tau\left(\frac{R}{K}\right)^{\frac{2}{3}}\left(F_{\mathrm{L}}+4 \pi \gamma R\right)^{\frac{2}{3}}
$$

Here $\tau$ is the shear stress in the friction contact, $\gamma$ the surface energy and $F_{\mathrm{Ad}}=4 \pi \gamma R$ the adhesion force in the DMT-Model. The normal force in this model is the sum of applied normal load $F_{\mathrm{L}}$ and the adhesion force $F_{\mathrm{Ad}}$.

The dependence of friction force on the applied normal load is shown in Figure 4 for the three distinct friction regimes (as was the case in previous figures). The intercept on the load axis as well as the values for the friction forces change from region (a) to (c). To extract more information the measured curves were fitted using Equ. 3 and the surface energy and the shear stress were then evaluated (see Fig. 5). Again a value of $K=50.1 \mathrm{GPa}$ was used and $R=30 \mathrm{~nm}$ was taken as the tip radius. This is larger than the manufacture-quoted tip radius (less than $15 \mathrm{~nm}$ ) because our studies indicate that the tip gets blunter during measurement $[12,4]$. The surface energy, $\gamma$, and the shear stress, $\tau$, (Fig. 5) reduce in two steps during pump-down. The three distinct pressure regimes, however, are still visible. 


\section{Discussion}

The dependence of friction on the sliding velocity and applied normal load for a silicon tip sliding against a silicon sample changes considerably from wet at air pressure to very dry in ultrahigh vacuum. The nanofriction between two hydrophilic surfaces (Fig. 1) is reduced in three regimes. The slope in the friction force vs. logarithm of sliding velocity curves (Fig. 3) describes the friction behavior of the nanotribological contact. The negative slope in regime (a) shows the domination of capillary forces. The friction force is determined by the number of capillary bridges that form in the nanocontact between tip and sample [15]. The number of capillary necks and the influence of capillary force are reduced at higher sliding velocities assuming an activation process for the time to build the water bridges [15]. When the water film thickness is above $2 \mathrm{~nm}$ [13], for tribological purposes water can be considered as bulk water. During pump-down, the slope changes to positive values after reduction of the residual gas pressure (and with that the water partial pressure). At this stage, bulk water desorbs when the water partial pressure of ambient is lower then the water vapor pressure in regime (b). The residual water film remaining on the sample surface, has a film thickness of about $0.7 \mathrm{~nm}$ and correlates to a film composed of 2 ice-like water bilayers $[13,8]$. In this regime, ordering effects of the ice-like bilayers strongly influence friction. We speculate that friction arises from the "pushing aside" of these bilayers by the sliding tip, which has always some solid-solid contact present due to the high applied normal load (pressure) in this study [13]. These ordering effects result in the positive slope observed in Fig. 2. The decreasing of the slope in region (c) is attributed to the complete removal of water due to desorption. A similar small positive slope was also measured on hydrophobic $[9,15,16]$ as well as on atomically flat [6] surfaces. Thus, our measurements demonstrate the transition of friction from a wet, hydrophilic surface to a dry hydrophilic one. From these results one can deduce that the characteristics of a dry hydrophilic surface are comparable to those of hydrophobic surfaces.

The frictional behavior derived from the nanocontact conditions is defined by the structure of the water film present on the surface. The water film in regime (a) is bulk water on top and an electrochemical double layer between this bulk water and silicon oxide surface [16]. A comparison between bulk water in regime (a) and ordered water in (b) (Figure 2) shows that, when an extrapolation of the friction force vs. sliding velocity curves in regime (a) and (b) is made to higher velocities, they cross at a velocity of about $2 \mathrm{~mm} / \mathrm{s}$. This result suggests that at this velocity the influence of capillary force vanishes and friction is 
dominated by ordering effects of the thin water layer. In other words, this means that the slope of the friction vs. logarithm of sliding velocity should reverse at velocities in the millimeter per second range for the examined contact parameters. However, since the highest achievable sliding velocity in the used device is about $14 \mu \mathrm{m} / \mathrm{s}$, experimental verification of this phenomenon was not possible.

According to the DMT-model [2], the surface energy relates to the adhesion force as $F_{\mathrm{Ad}}=4 \pi \gamma R$. Following this equation, adhesion increases for higher surface energies. In our experiments, the surface energy undergoes a reduction in 3 regimes (Fig. 5). The difference of surface energy between regime (a) and (b) is about $122 \mathrm{~mJ} \cdot \mathrm{m}^{-2}$ and is in the order of magnitude of the surface tension of bulk water $\left(\gamma_{\mathrm{H}_{2} \mathrm{O}}=72 \mathrm{~mJ} \cdot \mathrm{m}^{-2}\right)$. This confirms that bulk water desorbs during the transition from regime (a) to (b). The lowest value of surface energy is reached at pressures lower then $10^{-7}$ mbar, where it has a value of $21 \mathrm{~mJ} \cdot \mathrm{m}^{-2}$. This value is comparable to measurements determined by adhesion occurring in a micro cantilever array [10]. Another quantity, the shear stress, describes the lateral force per unit area for two moving parts in contact. In our experiments, the shear stress changes in two steps (Fig. 5). The highest shear stress is measured at high water film thicknesses in regime (a). At this stage, the effects of solid-solid contact, ordering of the electrochemical double layer and bulk water, resist the motion of the tip. The removal of the bulk water layer shows decreasing of shear stress in regime (b). At this stage, interactions due to the solid-solid contact and the ordering effects of the two ice-like water bilayers dictate the frictional properties. The lowest shear force (visible in regime (c)) is present when only solid-solid contact occurs.

It should be noted that at the applied normal load in this study, solid-solid contact also occurs in the regime where ordering effects of thin water double layers are present. Also, these two effects occur in the capillary force regime where bulk water is present on the surface.

\section{Conclusion}

The friction force was examined as a function of the residual gas pressure, sliding velocity and applied normal load using contact SFM. The friction force on hydrophilic silicon undergoes changes during pumpdown in a vacuum system from air to $10^{-8}$ mbar. Three distinct friction regimes with different nanofriction mechanisms and nanocontact characteristics, were identified. These regimes are shown schematically in Figure 6. First, the capillary force dominates. Later, during pumpdown, most of the water is removed by vacuum desorption. The re- 
maining water is now an ordered water double layer and the effects of the ordered water layer dominate friction. After complete water desorption due to the combined effects of the vacuum and frictioninduced desorption, only solid-solid contact remains, which exhibits the lowest friction of all the three friction regimes. In this study, the water present on the tip and sample surface never acts as a lubricant.

\section{Acknowledgements}

This work was supported by a grant from the Deutsche Forschungsgemeinschaft (Project Sche 425/2-4).

\section{References}

1. Bowden, F. P. and D. Tabor: 1950, The friction and lubrication of solids. Oxford: Clarendon Press.

2. Derjaguin, B. V., V. M. Muller, and Y. P. Toporov: 1975, 'Effect of contact deformation on the adhesion particles'. J. Colloid Interface Sci. 53, 314.

3. Deulin, E. A., A. A. Gatsenko, and B. A. Loginov: 1999, 'Friction force of smooth surfaces of $\mathrm{SiO}_{2}-\mathrm{SiO}_{2}$ as a function of residual pressure'. Surf. Sci. 433-435, 288.

4. Franzka, S.: 1999, 'Rasterkraftmikroskopische Untersuchungen zur Reibung von Silicium- und $\mathrm{Al}_{2} \mathrm{O}_{3}$-Einkristalloberflächen im Kontakt mit Silicium'. Dissertation, Universität Karlsruhe.

5. Gellman, A. J.: 1992, 'Lubrication by molecular monolayers at Ni-Ni interfaces'. J. Vac. Sci. Technol. A 10, 180.

6. Gnecco, E., R. Bennewitz, T. Gyalog, C. Loppacher, M. Bammerlin, E. Meyer, and H. Güntherodt: 2000, 'Velocity Dependence of Atomic Friction'. Phys. Rev. Lett. 84, 1172.

7. Iler, R. K.: 1979, The Chemistry of Silica. Chichester: Wiley Interscience.

8. Israelchvili, J. N. and R. M. Pashley: 1983, 'Molecular layering of water at surfaces and origin of repulsive hydration forces'. Nature 306, 249.

9. Liu, H., S. I.-U. Ahmed, and M. Scherge: 2001, 'Microtribological properties of silicon and silicon coated with diamond like carbon, octadecyltrichlorosilan and stearic acid cadmium salt films: A comparative study'. Thin solid films 381, 135.

10. Maboudian, R.: 1998, 'Surface processes in MEMS technology'. Surf. Sci. Rep. 30, 207.

11. Maugis, D.: 1992, 'Adhesion of Spheres: The JKR-DMT Transition Using a Dugdale Model'. J. Colloid Interface Sci. 150, 243.

12. McGuiggan, P. M., J. Zhang, and S. M. Hsu: 2001, 'Comparsion of friction measurements using the atomic force microscope and the surface forces apparatus: the issue of scale'. Tribol. Lett. 10, 217.

13. Opitz, A., S. I.-U. Ahmed, J. A. Schaefer, and M. Scherge: 2002, 'Friction of thin water films: a nanotribological study'. Surf. Sci. 504, 199. 
14. Opitz, A., S. I.-U. Ahmed, J. A. Schaefer, and M. Scherge: 2003, 'Nanofriction of silicon oxide surfaces covered with thin water films'. WEAR 254, 924.

15. Riedo, E., F. Lèvy, and H. Brune: 2002, 'Kinetics of Capillary Condensation in Nanoscopic Sliding Friction'. Phys. Rev. Lett. 88, 185505.

16. Scherge, M. and S. N. Gorb: 2001, Biological Micro- and Nanotribology, NanoScience and Technology. Berlin: Springer-Verlag.

17. Scherge, M., X. Li, and J. A. Schaefer: 1999, 'The effect of water on friction of MEMS'. Tribol. Lett. 6, 215.

18. Schwarz, U. D., P. Köster, and R. Wiesendanger: 1996, 'Quantitative analysis of lateral force microscopy experiments'. Rev. Sci. Instrum. 67, 2560.

19. Tanner, D. M., J. A. Walraven, L. W. Irwin, M. T. Dugger, N. F. Smith, W. P. Eaton, W. M. Miller, and S. L. Miller: 1999, 'The Effect of Humidity on the Reliability of a Surface Micromachined Microengine'. IEEE Int. Rel. Phys. Symp. Proc. p. 189. 
Nanofriction mechanisms derived from the dependence on load and sliding velocity 11

\section{Figure captions}

Figure 1.

Friction force vs. residual gas pressure during pump down in the vacuum chamber. The ranges (a), (b) and (c) identify different friction regimes. The solid line is shown to guide the eye.

Figure 2.

Friction force vs. sliding velocity for the three friction regimes identified in Fig. 1. For every friction regime a representative measurement is shown. The solid lines are fits using Equation 1.

Figure 3.

Slope $F_{\mathrm{S}}$ from Equation 1 vs. the residual gas pressure. The three different regimes from Fig. 1 are marked using the vertical grey lines. They correspond well with the change in the slope $F_{\mathrm{S}}$. The solid line connecting the data points has been drawn to guide the eye.

Figure 4.

Friction force vs. applied normal load for the three friction regimes from Fig. 1. A representative measurement is shown for each regime. The solid lines are fits using Equation 3. A zoomed diagram is shown for regime (c) for better visibility.

\section{Figure 5.}

Surface energy and shear stress as determined from Equation 3 vs. the residual gas pressure. The three different friction regimes from Fig. 1 are marked using the vertical grey lines. The solid line connecting the data points has been drawn to guide the eye.

Figure 6 .

Schematic model of water coverage in various coverage regimes determined by the friction behavior. While there is only the solid-solid interaction in regime (c), this interaction along with ordering effects of water are both present in regime (b) and, similarly, the solid-solid interaction and ordering effects are also present in regime (a). 


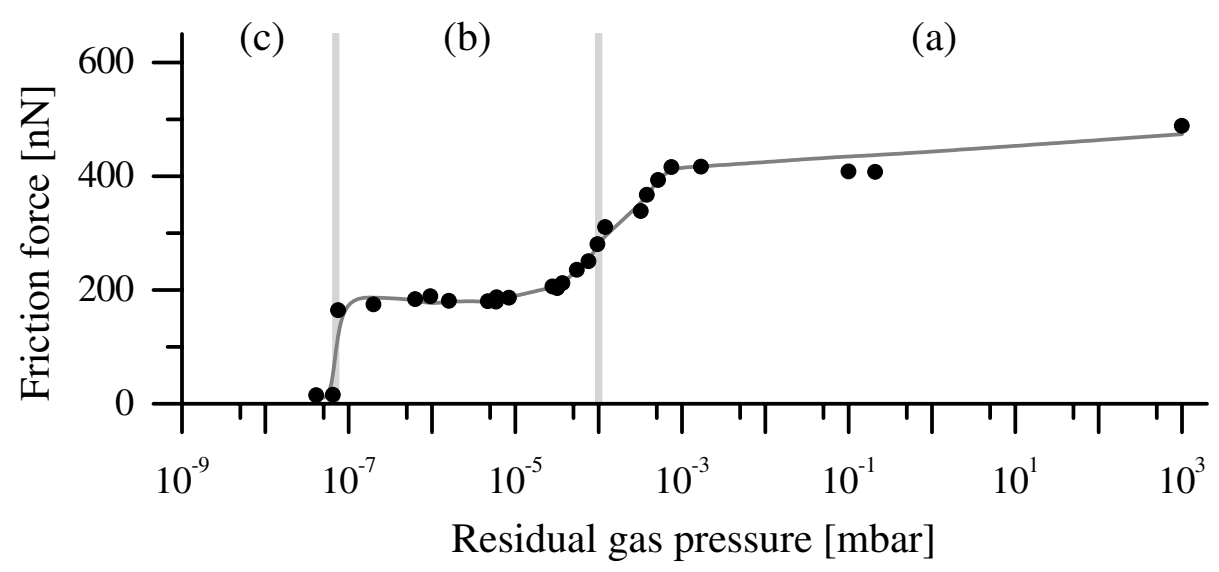

Figure 1.

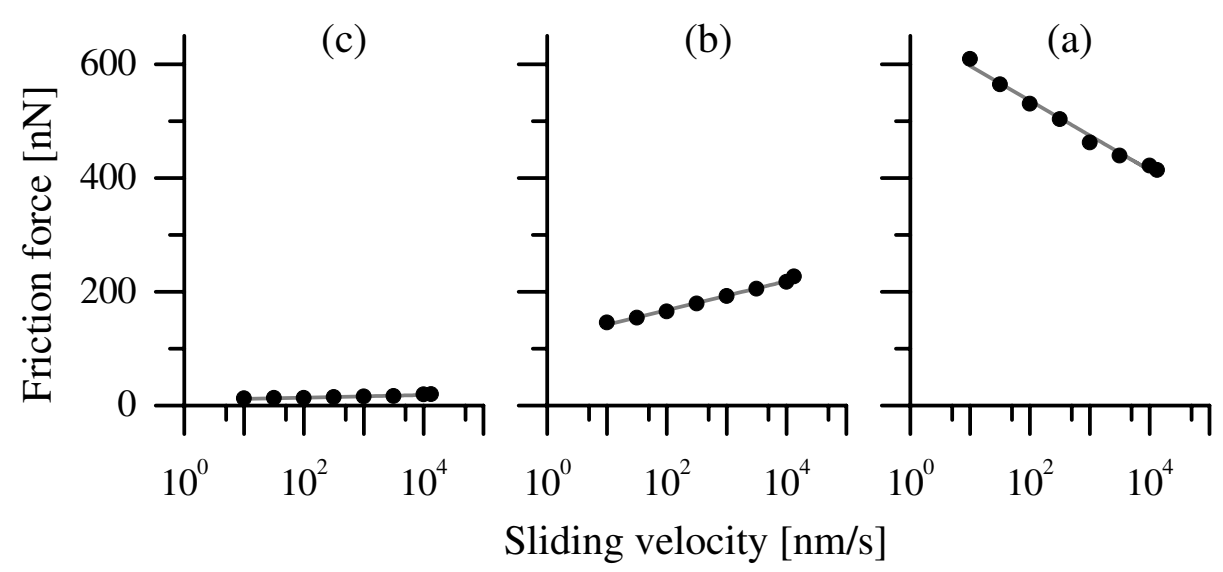

Figure 2. 


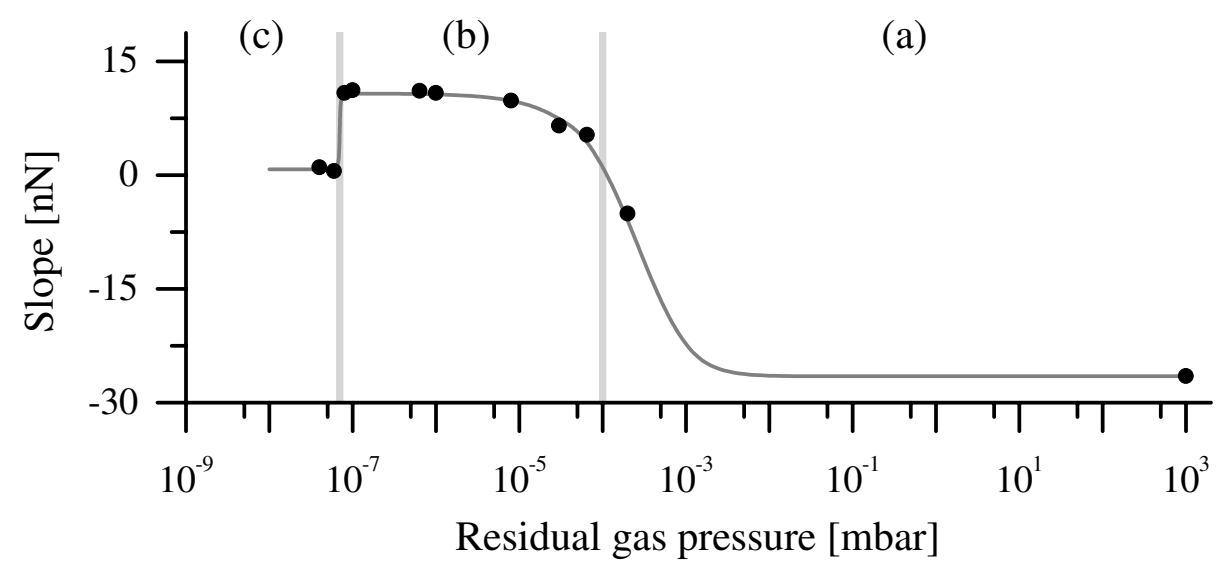

Figure 3.

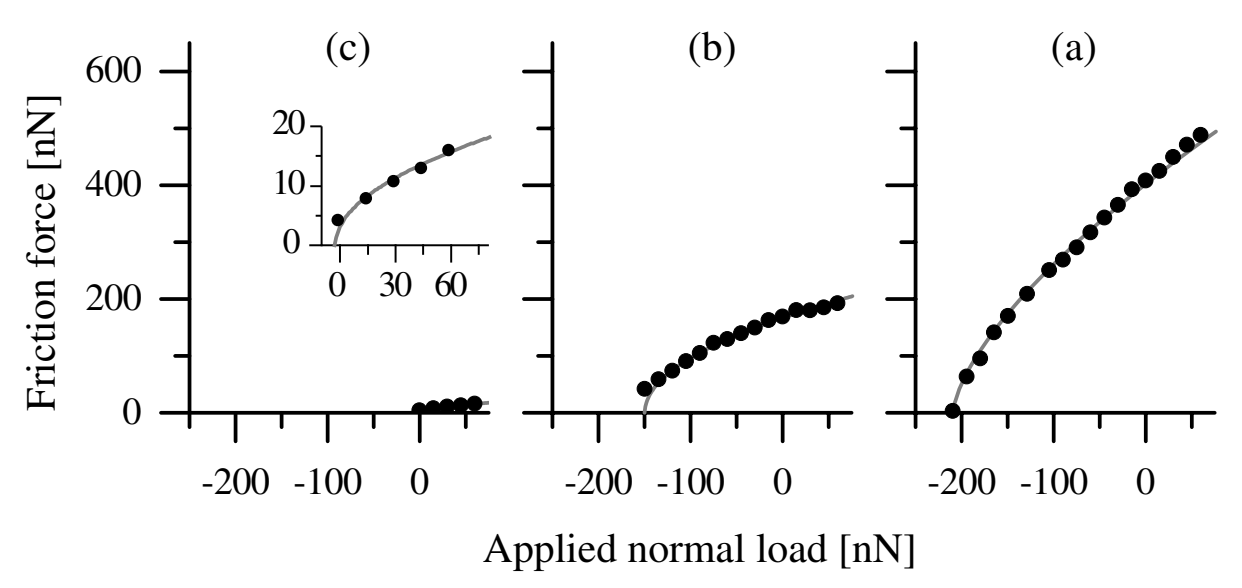

Figure 4. 


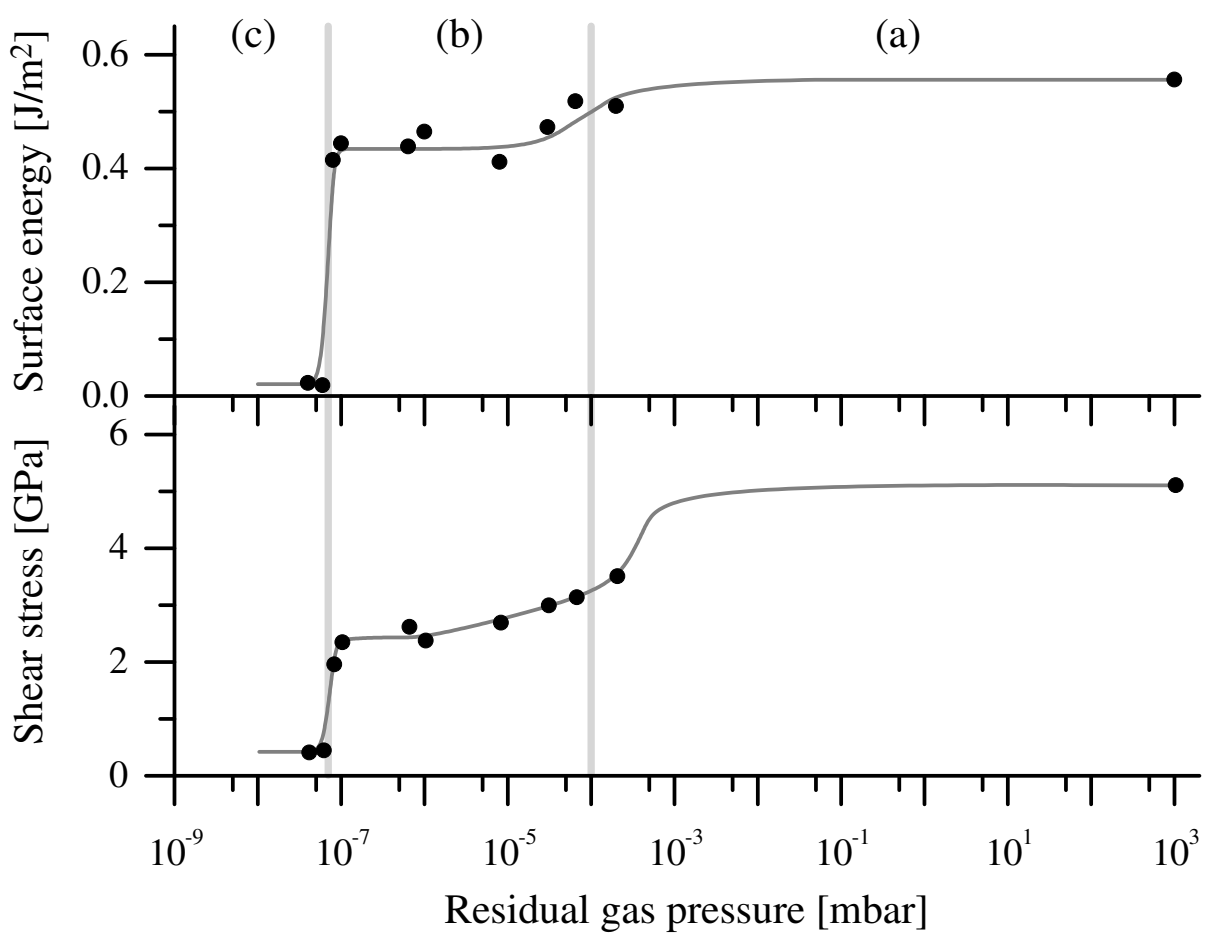

Figure 5.

(c)

solid-solid contact

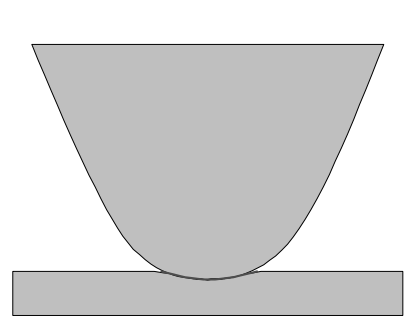

Figure 6 . (b) ordering effects dominate

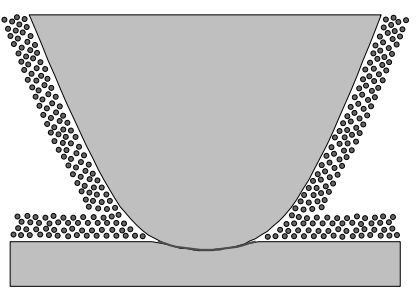

(a) capillary force dominates

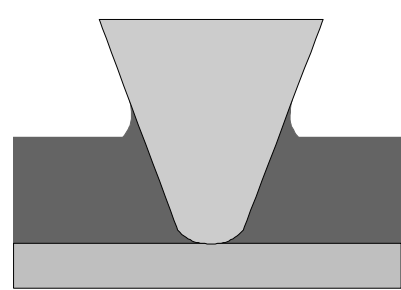

